\title{
9 \\ Physikalistische Graphologie als Avantgarde der Psychologie oder Physikalismus auf Abwegen
}

\author{
Thomas Mormann
}

\subsection{Carnaps Programm des Physikalismus}

Die Physikalisierung der Psychologie ${ }^{1}$ war für Carnap Teil eines Programms, das darauf zielte, die vermeintlich privilegierte Stellung des Menschen in der Welt als Illusion zu entlarven. Mit diesem Programm sah Carnap sich in der Ahnenreihe von Kopernikus, Darwin, Marx und Freud: Kopernikus habe gezeigt, dass die Erde als Wohnort der Menschheit nicht im Zentrum des Universums stehe, Darwin, dass der Mensch nur ein Tier unter anderen sei, Marx, dass die menschliche Geschichte weniger durch Ideen als durch materielle Tatsachen bestimmt werde, Nietzsche, dass moralische Werte eine biologische Fundierung haben, und Freuds Psychoanalyse habe deutlich gemacht, dass das menschliche Bewusstsein wesentlich durch unbewusste Triebkräfte bestimmt werde. ${ }^{2}$ Carnap schließlich war es darum zu tun, die These von der vermeintlichen Sonderstellung der Psychologie als Wissenschaft, welche sich angeblich aus der spezifischen Eigenart ihrer Thematik als Wissenschaft

\footnotetext{
${ }^{1}$ Cf. Psychologie in physikalischer Sprache (Carnap 1932/1933, S. 107-142).

${ }^{2}$ Siehe auch Freuds Eine Schwierigkeit der Psychoanalyse (1917). Dort zählte Freud eine Reihe von „Kränkungen“ auf, die die neuzeitlichen Wissenschaften dem Narzissmus des abendländischen Menschen zugefügt hätten. Freud erwähnt Kopernikus' heliozentrische Astronomie, Darwins Evolutionstheorie und als Letztes die Psychoanalyse. Diese sei als „Kränkung“ erfahren worden, da sie die Illusion zerstört habe, ,,das Ich wäre Herr im eigenen Hause“. Stattdessen habe sie gezeigt, dass das Seelenleben des Menschen weitgehend durch unbewusste Kräfte bestimmt werde, die der Kontrolle des Ich entzogen seien.
}

\footnotetext{
T. Mormann ( $\square)$

University of the Basque Country, Donostia-San Sebastian, Spanien

E-Mail: thomasarnold.mormann@ehu.eus

(C) The Author(s) 2021

C. Damböck, G. Wolters (eds.), Der junge Carnap in historischem Kontext:

1918-1935 / Young Carnap in an Historical Context: 1918-1935,

Veröffentlichungen des Instituts Wiener Kreis 30,

https://doi.org/10.1007/978-3-030-58251-7_9
} 
des menschlichen Fühlens und Denkens ergab, zu widerlegen und zu zeigen, dass die Psychologie als ein Teilgebiet einer idealen Physik aufgefasst werden könnte.

Ein solcher Physikalismus war für ihn eine Konsequenz einer allgemeinen wissenschaftlichen Einstellung, die diese Art von Aufklärung heroisch in Angriff nahm. Die Ablehnung einer solchen Physikalisierung interpretierte er, ähnlich wie Freud die Ablehnung der Psychoanalyse, als Ausdruck eines gekränkten Narzissmus, der zwar menschlich verständlich, aber wissenschaftlich ungerechtfertigt sei.

In etwas anderer Motivation zielte Carnaps Physikalismus darüber hinaus auch auf eine Überwindung der Trennung zwischen Geisteswissenschaften und Naturwissenschaften: Wenn die Psychologie sich als physikalisierbar erwies, also als Teil der Physik aufgefasst werden konnte, dann galt das a fortiori für die anderen vermeintlichen „Geisteswissenschaften“ wie Soziologie und Geschichtswissenschaft. Als Konsequenz würde sich ergeben, dass jede Wissenschaft als ein Zweig der Physik zu begreifen sei. Damit wäre auch das große Projekt des Wiener Kreises, nämlich die Vereinheitlichung der Wissenschaften in Gestalt einer enzyklopädischen „Einheitswissenschaft“, seiner Verwirklichung nahegekommen. Das schon allzu lange anhaltende Zeitalter der narzisstischen Illusion, wonach der Mensch in irgendeiner Weise etwas Besonderes, die Natur Transzendierendes sei, wäre damit zumindest für Menschen mit einer wissenschaftlichen Einstellung an sein Ende gekommen. Wer dann noch die Argumente für die Physikalisierbarkeit allen Wissens ablehne, zeige damit nur seine unwissenschaftliche, metaphysische Grundeinstellung.

Die These, dass die Physik die Grundwissenschaft sei, ist genauer so zu verstehen:

Wir wollen unter Physik nicht das System der heute bekannten physikalischen Gesetze verstehen, sondern die Wissenschaft, die durch die Art der Begriffsbildung gekennzeichnet ist: jeder Begriff geht zurück auf die „Zustandsgrößen“, das sind Zuordnungen von Zahlen zu Raumzeitpunkten nach bestimmtem Verfahren. Dann können wir unsere These, eine Teilthese des Physikalismus, so fassen: die Psychologie ist ein Zweig der Physik. (Carnap 1931, S. 142)

Wie diese auf „Zustandsgrößen“ fußende Begriffsbildung im Einzelnen funktionierte, brauchte nach Carnap den Wissenschaftsphilosophen zunächst nicht zu interessieren. Das zu untersuchen sei Sache des Einzelwissenschaftlers. Physikalisierung einer Wissenschaft war also für Carnap durchaus keine rein wissenschaftsphilosophische Angelegenheit, sondern eine arbeitsteilige Unternehmung, an der die Philosophie und die jeweils physikalistisch zu reformulierenden Wissenschaften beteiligt waren. Es war Aufgabe der Wissenschaftler, ihre Wissenschaft in ein für die Physikalisierung geeignetes Format zu bringen und so für die von den Philosophen im Einzelnen durchzuführende physikalistische Reformulierung vorzubereiten.

Carnaps Argument für die Physikalisierbarkeit der Psychologie war - zurückhaltend formuliert - ziemlich eigenartig. Es basierte auf der These der Physikalisierbarkeit 
der Graphologie $e^{3}$ als einer zentralen Teildisziplin der Psychologie. ${ }^{4}$ Der frühe Carnap war also nicht nur überzeugt, die Graphologie wäre eine wissenschaftlich respektable Disziplin; er vertrat darüber hinaus sogar die These, die Graphologie wäre der begrifflich am weitesten fortgeschrittene und deshalb am leichtesten physikalisierbare Teil der Psychologie. Überdies komme dem Nachweis, dass die Psychologie in die physikalistische Universalsprache übersetzt werden könne, eine besondere strategische Bedeutung deswegen zu, weil die Psychologie üblicherweise als die am schwierigsten zu erobernde Bastion des Antiphysikalismus angesehen werde: Über die prinzipielle Physikalisierbarkeit der Chemie, der Biologie und der anderen empirischen Wissenschaften sei man sich ja weitgehend einig. Falle auch die Psychologie der Physikalisierung anheim, stehe einer durchgehenden Physikalisierung aller Wissenschaften grundsätzlich nichts mehr im Wege.

Die Physikalisierung einer Wissenschaft setzt zunächst einmal voraus, dass ihre Begriffe klar und präzise formuliert sind. Wie Carnap bedauernd feststellt, sei das für die zeitgenössische Psychologie leider nur teilweise der Fall. Deshalb sei es zweckmäßig, das Physikalisierungsprojekt zunächst auf ein Teilgebiet der Psychologie zu beschränken, das bereits über einen hinreichend präzisen Begriffsapparat verfüge. Das sei, so behauptete er, die Graphologie. Dank der wegweisenden Arbeiten Ludwig Klages' sei sie der wissenschaftlich am weitesten fortgeschrittene Teil der Psychologie, deren vollständige und explizite Physikalisierung damit ohne allzu große Schwierigkeiten durchführbar sei.

Carnaps Projekt sei im Folgenden kurz als „Graphologieprojekt“ bezeichnet. Der Graphologie-Episode in Carnaps philosophischer Entwicklung ist bisher nur geringe bis gar keine Aufmerksamkeit geschenkt worden. Gleichwohl bin ich der Auffassung, dass

\footnotetext{
${ }^{3}$ Carnaps These, die Physikalisierung der Psychologie insgesamt werde durch die Physikalisierung der Graphologie als einer ,strategischen“ Teildisziplin nahegelegt, weist eine gewisse Parallele mit Freuds These auf, der zufolge die Physikalisierung der Psychologie insgesamt durch die Tatsache belegt werde, dass die Psychoanalyse als eine „normale“ Naturwissenschaft im Sinne der Physik konstituiert werden könne. Erst dadurch erweise sich die Psychologie insgesamt als eine „richtige“ Wissenschaft. Noch in einer nicht vollendeten, erst posthum veröffentlichten Arbeit behauptete Freud: „Unsere Annahme eines räumlich ausgedehnten, zweckmässig zusammengesetzten, durch die Bedürfnisse des Lebens entwickelten psychischen Apparates [...] hat uns in den Stand gesetzt, die Psychoanalyse auf einer ähnlichen Grundlage aufzurichten wie jede andere Naturwissenschaft, z. B. wie die Physik" (Freud 1940, 58). Freuds These des naturwissenschaftlichen Charakters der Psychoanalyse hat eine bis heute anhaltende lebhafte Diskussion ausgelöst. Von einigen wird sie als „,szientistisches Selbstmißverständnis“ der Psychoanalyse kritisiert (siehe Habermas 2001, 301). Carnaps Projekt der Physikalisierung der Graphologie ist, soweit ich weiß, nirgendwo auf Widerhall gestoßen.

${ }^{4}$ Heute wird die Graphologie wie die Astrologie, die Homöopathie, die Parapsychologie, der Kreationismus und die Psychoanalyse von den meisten Vertretern einer an Wissenschaftlichkeit und Rationalität orientierten Philosophie als Pseudo- oder Scheinwissenschaft betrachtet. Das schließt natürlich nicht aus, dass diese Disziplinen für die Wissenschaftsphilosophie von Interesse sind. Sie werfen die Frage auf, warum es sich bei ihnen um Pseudowissenschaften handelt. Allgemeiner stellt sich die Frage, ob es überhaupt verlässliche Kennzeichen gibt, Wissenschaft und Pseudowissenschaft voneinander zu unterscheiden.
} 
dieses Projekt mehr ist als ein Kuriosum. Zwar hat Carnaps wissenschaftsphilosophische Auseinandersetzung mit der Graphologie nach 1932/1933 keine Fortsetzung gefunden, ich möchte aber behaupten, dass sich im Graphologieprojekt der allgemeine Stil des Carnapschen Philosophierens besonders deutlich manifestiert, nämlich von einer sehr abstrakten und idealisierten Vorstellung einer Wissenschaft auszugehen, aus der dann weitreichende philosophische Folgerungen gezogen werden, die nur schwer mit der tatsächlichen Praxis dieser Disziplin in Einklang zu bringen sind.

Im Einzelnen ist dieses Kapitel wie folgt gegliedert: Zunächst soll in Abschn. 2 Carnaps These, die Graphologie sei die Avantgarde einer wissenschaftlichen, ,physikalisierten“ Psychologie, genauer erörtert werden. Das Verdienst, den Weg zu einer Physikalisierung der Graphologie und damit zu ihrer vollständigen Verwissenschaftlichung geebnet zu haben, schreibt Carnap Ludwig Klages zu, auf dessen Buch Handschrift und Charakter (Klages 1989 [1917]) er sich in den graphologischen Abschnitten von „Psychologie in physikalischer Sprache“(Carnap 1931) explizit bezieht. In Abschn. 3 wird Carnaps Skizze einer physikalistischen Konstitution psychologischer Eigenschaften vorgestellt. Dann sollen in Abschn. 4 einige typische graphologische "Charakterbilder" verschiedener Grafologen (Klages, Christiansen und Elisabeth Carnap) vorgestellt werden. Diese belegen, dass Carnaps Vorstellungen von Graphologie als einer Wissenschaft eine beträchtliche Distanz zur graphologischen Praxis aufweisen.

Wie allgemein bekannt, war Klages nicht nur einer der Gründerväter der modernen Graphologie, er war auch einer der einflussreichsten deutschen Intellektuellen in der Zeit des späten Wilhelminismus und der Weimarer Republik. Bei der Verbreitung irrationalistischer, präfaschistischer und antisemitischer Ideologeme, insbesondere in der deutschen Jugendbewegung, spielte er zu Anfang des 20. Jahrhunderts eine fatale Rolle. Darauf ist Carnap nie eingegangen. Gleichwohl hat er Klages nicht nur als Graphologen, sondern auch als Philosophen zur Kenntnis genommen. Insbesondere Klages' monumentales Hauptwerk Der Geist als Widersacher der Seele (Klages 1981 [1929-1932]) hat ihn offenbar nachhaltig beeindruckt. Auf Klages' Rolle in der deutschen philosophischen Szene soll deshalb in Abschn. 5 genauer eingegangen werden. Wissenschaftsphilosophische Debatten um den Physikalismus sind bis heute ein zentrales Thema der Wissenschaftsphilosophie, auch wenn der heute diskutierte Begriff von Physikalismus wenig mit dem zu tun hat, den Carnap in den 1930er-Jahren erörterte. In Abschn. 6 möchte ich daher nur auf die Kritik an Carnaps Physikalismus, die Cassirer in den 1930er- und 1940er-Jahren vorbrachte, eingehen. Schließlich soll im letzten Abschn. 7 expliziert werden, in welchem Sinne die Idee einer physikalisierten Graphologie als typisches Artefakt einer carnapianischen Wissenschaftsphilosophie verstanden werden kann.

\subsection{Graphologie als Avantgarde der wissenschaftlichen Psychologie?}

Zunächst werde etwas genauer erklärt, warum Carnap die Graphologie als das wissenschaftlich am weitesten fortgeschrittene Teilgebiet der Psychologie ansah. Sein Argument für diese Behauptung war folgendes: 
Merkwürdigerweise hat nun die Physikalisierung bemerkenswerte Erfolge auf einem Teilgebiet der Psychologie aufzuweisen, das noch vor verhältnismäßig kurzer Zeit mit rein intuitiver (oder höchstens pseudo-rationaler) Methode und aufgrund völlig unzulänglicher Empirie betrieben wurde und daher überhaupt noch keinen Anspruch auf Wissenschaftlichkeit erheben konnte, nämlich auf dem Gebiet der Graphologie. Die theoretische Graphologie, von der hier allein die Rede sein soll, untersucht die gesetzmäßigen Zusammenhänge zwischen Gestalteigenschaften der Schriftzüge und denjenigen psychologischen Eigenschaften des Schreibenden, die man als „Charaktereigenschaften“ zu bezeichnen pflegt. (Carnap 1932/1933, S. 130)

Carnap behandelte Klages' „Methode“, Charaktereigenschaften des Schreibers aus geometrischen Eigenschaften seiner Schrift abzuleiten, als eine Art Blackbox, deren Inneres ihn als Philosophen nicht zu interessieren braucht. Dieser Ansatz ähnelt, wie er selbst ausführt, der (antiquierten) Verwendung eines Laubfrosches zur Wettervorhersage oder, etwas moderner, der Verwendung eines „Diagnosehundes“ Beispiele, die Carnap in „Psychologie in physikalischer Sprache“ ausführlich diskutierte (Carnap 1932/1933, S. 128). Ein solcher Hund hat die Fähigkeit, das Vorliegen gewisser Krankheiten riechen zu können, ohne dass der Arzt wissen musste, wie das im Einzelnen funktioniert. Klages, oder besser seine ,ausdruckswissenschaftlich" begründete Graphologie, hatte für Carnap die Funktion eines solchen „metaphysisch-graphologischen ${ }^{5}$ Diagnosehundes“, der anhand von Schriftproben zutreffende Charakterbeschreibungen von Menschen lieferte, ohne dass dem Philosophen oder dem Psychologen klar sein musste, wie er das im Einzelnen anstellte. Die Verwendung von Laubfröschen und Diagnosehunden mag legitim sein, solange sie empirisch gute, nachprüfbare Resultate liefert. Das Problem ist nur, dass die graphologische Methode nach allgemeiner Auffassung (auch nach Auffassung der Graphologen) das nicht leistet und auch nicht leisten kann. Klages selbst wurde nicht müde, das zu betonen:

Endlich kann man sich nicht genug in der Überzeugung bestärken, daß es auf dem Gebiet der Seelendeutung keinerlei mechanisches Verfahren gibt. [...] Die Unterscheidungsfähigkeit für menschliche Charakterzüge hängt durchaus von der Breite charakterologischer Erfahrung und der Tiefe charakterologischen Wissens ab. (Klages 1989 [1917], vii)

Die Nichtphysikalisierbarkeit der Graphologie wurde nicht nur von Klages selbst behauptet, sie wird bis heute von praktisch allen Graphologen vertreten, um übertriebene, unrealistische Erwartungen an die Graphologie von vornherein zurückzuweisen:

Die Graphologie ist kein Testverfahren. Sie ist keine messende und zählende Methode; sie hat die gefundenen Merkmale nicht nur zu registrieren, sondern zu wägen, d. h. ihnen ihre Bedeutung und ihren Platz in der Handschrift zu geben [...] (Wittlich 1989, viii)

Wittlich als „moderner Graphologe“ widerspricht der Möglichkeit einer Physikalisierung der Graphologie also ganz unmissverständlich. Carnaps Projekt einer physikalisierten Graphologie hat auch bei den von der Wissenschaftlichkeit ihrer Disziplin überzeugten Graphologen kaum Widerhall gefunden, weil diese ein

\footnotetext{
${ }^{5}$ Diese auf den ersten Blick etwas seltsam anmutende Charakterisierung soll darauf hinweisen, daß Klages' graphologische Methoden eng mit seiner ausdruckswissenschaftlichen Metaphysik verknüpft waren.
} 
„weicheres“, eher an den hermeneutischen Wissenschaften orientiertes Modell ihrer „Wissenschaft“ favorisierten. Sie waren zwar davon überzeugt, ein guter Graphologe könnte aufgrund der Analyse von Schriftproben einer Person zuverlässige Aussagen über deren Charakter machen, sie begriffen die Graphologie aber eher als eine hermeneutische Disziplin, der es um die Auslegung graphologischer Zeugnisse zu tun ist. So charakterisiert Bernhard Wittlich, der Herausgeber neuerer Auflagen von Klages' graphologischem Hauptwerk Handschrift und Charakter, die Graphologie als ,ein Verfahren, das sich der Statistik, dem Messen und Zählen nicht recht fügen will““ (in Klages 1989 [1917], Geleitwort, x). Er bestreitet überdies explizit die Möglichkeit einer Carnapianischen Physikalisierung der Graphologie, deren Unmöglichkeit er durch das folgende Gedankenexperiment plausibel machen möchte:

\begin{abstract}
Wollte jemand von einem Graphologen die Deutung einer Handschrift verlangen, indem er diesem - etwa telefonisch - alle sachlichen Merkmale der betreffenden Handschrift nennen würde, also etwa ihre Größe, ihren Neigungswinkel, die Bindungsform, den Verbundenheitsgrad, die Längenunterschiede, die Weite und Breite, die Regelmäßigkeit usw., so wäre es völlig ausgeschlossen, danach ein auch nur einigermaßen zuverlässiges Charakterbild abzuleiten. Der Graphologe würde das Ganze, den Eindruckscharakter, den Gehalt, die Lebendigkeit dieser Schrift, nach Klages also ihren Rhythmus, ihr Formniveau, nicht aus den Merkmalen gewinnen oder ableiten können. [...] Er könnte also auch kein einziges dieser Merkmale deuten, jedes von ihnen bliebe nicht allein wegen der grundsätzlichen Doppeldeutigkeit, sondern auch noch an und für sich vieldeutig. (Klages 1989 [1917], Geleitwort, viii)
\end{abstract}

Carnap ist auf derartige Einwände gegen eine Physikalisierung der Graphologie niemals eingegangen. Das Argument, ihm wäre es eben nur um die Theorie der Graphologie gegangen und nicht um ihre Praxis, dürfte nicht alle überzeugen.

Auch bei den zahlreichen einer Physikalisierung der Wissenschaften nicht abgeneigten Philosophen ist Carnaps Projekt auf keine Sympathie gestoßen, einfach weil die Graphologie kaum je als ernst zu nehmende Wissenschaft angesehen wurde. Das Scheitern von Carnaps Projekt einer Physikalisierung der Graphologie impliziert natürlich nicht, dass das Programm einer Physikalisierung der Psychologie überhaupt gescheitert wäre. ${ }^{6}$ Carnaps Physikalismus spielt in der zeitgenössischen Debatte dieses Themas höchstens eine kleine Rolle; gleichwohl, so möchte ich behaupten, bleibt der in „Psychologie in physikalischer Sprache“ unternommene Versuch einer Physikalisierung der Graphologie ein interessantes Zeugnis Carnapscher Wissenschaftsphilosophie.

\title{
9.3 Physikalistische Konstitution psychologischer Eigenschaften
}

Carnap ging davon aus, dass psychologische (Charakter-)Eigenschaften von Personen in strikter Analogie zu physikalischen Eigenschaften physikalischer Systeme konstituiert werden können. Charaktereigenschaften seien, so Carnap, als Dispositionseigenschaften zu verstehen. So ist die Aussage , $M$ hat einen aufbrausenden Charakter" als

\footnotetext{
${ }^{6}$ Für eine Darstellung des modernen Physikalismus siehe Kim (2008).
} 
dispositionale Aussage der folgenden Art zu interpretieren: „, $M$ reagiert in den und den Situationen in einer Weise, die kompetente Beobachter als aufbrausend bezeichnen." Personen, deren Charakter wir als ,gelassen“, ,gleichmütig“ oder ähnlich beschreiben, tun dies hingegen nicht. Die Charaktereigenschaft ,aufbrausend“ kann damit analog zur physikalischen Eigenschaft ,elektrisch leitfähig“ beschrieben werden: Ein Stoff ist „elektrisch leitfähig“, wenn er in den und den Situationen in einer Weise reagiert, die kompetente Beobachter als das Leiten eines elektrischen Stromes beschreiben.

Nach Carnap existiert eine strikte Analogie zwischen der Konstitution physikalischer und psychologischer Dauereigenschaften aus Momentanaussagen. Ein Mensch, oder besser, das wahrnehmbare Verhalten eines Menschen wird also als Anzeiger gewisser psychischer Zustände aufgefasst, in denen sich dieser Mensch befindet. Nach Carnaps Verständnis von Psychologie befindet sich ein Mensch $M$ in jedem Augenblick seiner Existenz in bestimmten psychischen Zuständen, etwa , $M$ ist zornig“, „, $M$ ist gelassen“, „, $M$ ist fröhlich“, , $M$ ist aufgeregt“ usw. - analog einem physikalischen System $S$, das sich in jedem Augenblick in gewissen physikalischen Zuständen befindet, , $S$ hat die Temperatur $t^{\prime},{ }_{,} S$ erfährt die Beschleunigung

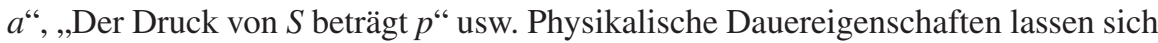
dann gewissermaßen als Integrale von Momentaneigenschaften verstehen: Ein Stoff $S$ ist leitfähig, wenn sich in bestimmten experimentellen Situationen gewisse Phänomene zeigen - eben dass er Strom leitet. Zeigen sich diese Phänomene nicht, wird $S$ als nicht leitfähig klassifiziert. Entsprechend ergeben sich für Carnap dauerhafte Charaktereigenschaften aus dem momentanen Verhalten einer Person (cf. Carnap 1932/1933, S. 112).

Eine physikalisierte Graphologie behauptet dann, dass eine enge Korrelation zwischen den Charaktereigenschaften und den Schrifteigenschaften besteht, dass man also von den Formeigenschaften der Schrift eines Menschen auf seine Charaktereigenschaften schließen könne. Es wird also behauptet, dass sich konstante Dispositionseigenschaften eines Menschen wie ,großzügig“, „undiszipliniert“, „gehemmt“ sich in gewissen Eigenschaften seiner Schriftzüge niederschlagen. Wie dieser Mechanismus im Einzelnen funktioniert, braucht den Wissenschaftsphilosophen nicht zu interessieren. Der entscheidende Punkt ist, dass der Mechanismus in zuverlässiger Weise funktioniert. Die Physikalisierung der Graphologie und das heißt die ultimative Verwissenschaftlichung der Graphologie als eines Teilgebietes der Psychologie, wäre erreicht, wenn die folgenden drei Aufgaben gelöst sind:

(1) Die graphologisch als relevant betrachteten stabilen Merkmale einer Schrift, die von Graphologen meist anschaulich oder metaphorisch als „schwungvoll“", „schwankend“, „,willensschwach“ usw. beschrieben werden, können rein geometrisch (,figural“" nach Carnap), etwa durch Verhältnis und Form ihrer „Bögen“, „Winkel“, ,Unterlängen“, „Oberlängen“ usw., beschrieben werden. ${ }^{7}$

\footnotetext{
${ }^{7}$ Klages bestreitet explizit, eine ,ausdruckswissenschaftliche“ grafologische Analyse einer Schriftprobe ginge so einfach vonstatten. Carnap geht darauf nicht ein, wohl weil er dies für ein Problem der ,praktischen“ "Graphologie hält, das ,,theoretisch irrelevant“ ist. An dieser Schwierigkeit hängt aber gerade die Empirizität der Klages'schen „Ausdruckswissenschaft“.
} 
(2) Die in den graphologischen Aussagen vorkommenden stabilen psychologischen Charaktereigenschaften (,ich-orientiert“, ,phantasievoll“) sind physikalistisch zu beschreiben.

(3) Eine zuverlässige statistische Korrelation zwischen (1) und (2) ist aufzustellen.

Carnap war der Auffassung, diese Aufgaben seien lösbar oder sogar, insbesondere durch Klages' wegweisende Erkenntnisse, bereits gelöst. Genauer kam er zu folgender Einschätzung:

Diese Aufgabe der Physikalisierung [also (1), TM] der Schrifteigenschaften ist von den Graphologen in vielen Fällen schon zum guten Teil gelöst worden. Vergleiche z. B. Ludwig Klages, Handschrift und Charakter. Mehrere unserer Beispiele sind diesem Buch entnommen oder in Anknüpfung an seine Darlegungen aufgestellt (ibid.). Gegen eine Beibehaltung der vom intuitiven Eindruck hergenommenen Bezeichnungen (z. B. „voll“, „,mager“, „schlank“, „wuchtig“ und dergl.) ist nichts einzuwenden; unserer Forderung ist Genüge getan, sobald für eine solche Bezeichnung eine Definition in rein figuralen Bestimmungen aufgestellt ist. (Carnap 1932/1933, S. 133)

Kurz, Carnap attestierte Klages, die Aufgabe (1) im Wesentlichen gelöst zu haben. Klages' „Lösung“ basiert auf der von ihm selbst inaugurierten „Ausdruckswissenschaft". 8

Die Lösung der Teilaufgabe (2) setzt Carnaps Meinung nach die Physikalisierbarkeit psychologischer Momentanaussagen voraus. Diese Aufgabe schätzt Carnap als schwierig, aber lösbar ein. Er schien dabei anzunehmen, dass dies in ähnlicher Weise möglich sei, wie man die von ihm als ,typisch psychologisch“ betrachtete Aussage , $A$ ist aufgeregt“ in die physikalistische Aussage „Der Leib des $A$ ist physikalisch-aufgeregt" übersetzen konnte. Carnap skizziert eine solche physikalistische Übersetzung so:

Der Leib des $A$ ist in einem Zustand, der dadurch gekennzeichnet ist, daß Frequenz von Atmung und Puls erhöht sind und auf gewisse Reize noch weiter erhöht werden, daß auf Fragen meist heftige und sachlich unbefriedigende Antworten gegeben werden, daß auf gewisse Reize hin erregte Bewegungen eintreten und dergl. (Carnap 1932/1933, S. 115/116)

Selbst wenn man diese Skizze einer physikalistischen Übersetzung von ,A ist aufgeregt“" als erste Phase einer physikalistischen Übersetzung akzeptiert, die im Prinzip zu einer vollständig physikalisierten Aussage ergänzt werden könnte, bleibt zweifelhaft, ob ein Satz wie „A ist aufgeregt“ als typisch für die Sätze angesehen

\footnotetext{
${ }^{8}$ Diese ,ausdruckswissenschaftliche Lösung“ war allerdings wesentlich komplizierter, als Carnap zur Kenntnis nahm. Bevor ein genaues Merkmalsprotokoll der zu analysierenden Schrift aufgenommen wurde, gewann Klages einen allgemeinen Eindruck vom „Formniveau“ der Schrift, d. h. deren Rhythmus, Ebenmaß, Regelmäßigkeit, Geübtheit, Eigenständigkeit und charakteristischer Ausbildung. Er unterschied fünf verschiedene Formniveaus. Die Beurteilung des Formniveaus lieferte den allgemeinen Rahmen für jede Schriftanalyse. Einzelne Schriftmerkmale waren immer mehrdeutig und mussten nach einem umfangreichen System von Prinzipien und Regeln interpretiert werden. Alle Schriftmerkmale konnten positiv oder negativ interpretiert werden. Welche Interpretation als gültig angesehen wurde, hing ab vom Einfühlungsvermögen und der Erfahrung des Graphologen. Carnap sagte nichts über diese zentralen Begriffe der Klages'schen Graphologie wie „Formniveau“ und „Rhythmus“.
} 
werden kann, die in graphologischen Charakterbeschreibungen à la Klages tatsächlich vorkommen. Beispiele, die einen solchen Zweifel erhärten, werden im nächsten Abschn. 4 genauer diskutiert werden.

Die Teilaufgabe (3) schließlich, die auf die Erstellung einer zuverlässigen Korrelation zwischen Aussagen über Schriftproben und Charakteraussagen hinauslief, hielt Carnap für eine Routineangelegenheit. Sie bestand darin, die zunächst intuitiv gewonnene Einsicht in die Zusammengehörigkeit einer Schrifteigenschaft mit einer Charaktereigenschaft durch statistisch gesicherte Vergleiche zu bestätigen. Das hätte zur Konsequenz, dass zwei kompetente Graphologen, die dieselbe Schriftprobe beurteilen, im Wesentlichen zum selben Ergebnis kommen müssten. Klages hielt das für einen Irrtum. Ein graphologisches Gutachten sei vielmehr eine Art künstlerisches Portrait einer Person, die das „Wesen“ dieser Person zum Vorschein bringe. Von einem Portrait ein und derselben Person erwarte man ja auch nicht, dass zwei eigenständige Künstler zum selben Ergebnis kommen würden. Das einzige, was man erwarten könne, sei, dass die Gutachten zweier kompetenter Graphologen sich nicht widersprächen. Carnaps physikalisierte Graphologie hingegen insistierte auf statistischen Korrelationen, d.h. analog zu den klassischen Naturwissenschaften sollten zwei Graphologen unter Berücksichtigung kleiner statistischer Abweichungen zu denselben Ergebnissen kommen.

Eine vollständige Physikalisierung der Graphologie liefe also hinaus auf eine vollständige Lösung von (1)-(3). Sie hätte Vorbildcharakter für die Psychologie insgesamt:

Unsere Auffassung geht nun dahin, daß für die gesamte Psychologie die Weiterentwicklung und Präzisierung der Begriffe in der Richtung vorzunehmen ist, die wir soeben am Beispiel der Graphologie angedeutet haben, also in der Richtung der Physikalisierung. (Carnap 1932/1933, S. 134)

Auch ohne eine derartige vollständige Physikalisierung wäre die Psychologie nach Carnaps Auffassung schon eine physikalische Wissenschaft, einfach deswegen, weil, wie er schlicht dekretiert, ihre Aufgabe darin bestehe, „das (physikalische) Verhalten der Lebewesen, insbesondere der Menschen, systematisch zu beschreiben und unter Gesetze zu bringen“ (Carnap 1932/1933, S. 134). Er weist also die Intentionen einer ,verstehenden Psychologie“ von vornherein ab. Die vollständige Physikalisierung im eben beschriebenen Sinne bedeute nur

eine höhere, stärker systematisierte wissenschaftliche Form der Begriffsbildung; ihre Durchführung ist eine praktische Aufgabe, die nicht mehr dem Erkenntnistheoretiker, sondern dem Psychologen zusteht. (Carnap 1932/1933, S. 135 f.)

Wie bereits gesagt, weder die praktizierenden Graphologen noch die an Graphologie interessierten Psychologen sind Carnap in dieser Einschätzung gefolgt.

Tatsächlich ist die von ihm behauptete Lösbarkeit der Probleme, die bei einer Physikalisierung der Graphologie auftreten, für die real existierende Graphologie völlig utopisch. Um das zu sehen, muss man nur einige Beispiele graphologischer 
„Charakterbilder" betrachten, wie sie von Klages und anderen als typisch für graphologische Erkenntnis angesehen wurden. Das geschieht im nächsten Abschnitt.

Zuvor sei noch einmal auf den arbeitsteiligen Charakter einer solchen Physikalisierung hingewiesen. Die Hauptlast der Physikalisierung bürdete Carnap den praktizierenden Graphologen auf: Die Philosophie formulierte nur theoretische Forderungen, deren praktische Realisierung anderen überlassen blieb. Das Problem der wissenschaftlichen Praxis war ein Problem, zu dem die Philosophie nichts zu sagen hatte. Eine philosophische Theorie wissenschaftlicher Praxis gab es für Carnap nicht. Der „Erkenntnistheoretiker“ sagt dem „Praktiker“, was er zu tun hat, um seine Wissenschaft auf ein höheres begriffliches Niveau zu bringen. Eventuelle Einwände, die den mangelnden Realitäts- und Praxisbezug der Vorstellungen des Theoretikers monierten, hatten für Carnap meist ein geringes Gewicht. Ihm ging es immer nur um eine Theorie der Wissenschaft.

Gleichgültig, wie man die Erfolgsaussichten einer Physikalisierung der Graphologie einschätzt, und auch unabhängig davon, ob Carnaps Darstellung der Graphologie die Realität dieser Disziplin trifft, ist es eine naheliegende Frage, was Carnap veranlasst haben mag, sich mit diesem, nach üblichen Maßstäben doch ziemlich abseitigen Gebiet relativ ausführlich zu befassen. Eine Antwort auf diese Frage ist in Carnaps Biographie zu finden.

Sein Interesse an der Graphologie wurde wohl durch seine Bekanntschaft mit Broder Christiansen (1869-1958) geweckt ${ }^{9}$ (cf. Steinfeld 2016, Kap. 7; Thomsen 2008). Christiansen hatte bei Rickert promoviert und lebte als Schriftsteller und Inhaber eines kleinen Verlages in Buchenbach in der Nähe von Freiburg. Carnap selbst war nach seiner Promotion in Jena mit seiner Frau und seinen Kindern nach Buchenbach gezogen, wo sein Schwiegervater ein Landgut besaß, und wohl auch, um seine Studien bei Husserl im nahen Freiburg fortzusetzen.

Um das Jahr 1930 zog Broder Christiansen mit Elisabeth Carnap, der ersten Frau Carnaps, zusammen, mit der er schon über mehrere Jahre ein von ihrem Ehemann akzeptiertes Verhältnis hatte. Elisabeth Carnap widmete sich mit Christiansen als ihrem Lehrer und Mentor der Graphologie. Zusammen veröffentlichten sie das

\footnotetext{
${ }^{9}$ Nach bürgerlichen Maßstäben war Christiansen eine recht seltsame Gestalt (zu Einzelheiten seiner Biographie und seines Werkes siehe Steinfeld 2016). In seinem Tagebuch erwähnt Carnap häufige Diskussionen mit Christiansen über graphologische und andere Themen. Ob man allerdings so weit gehen sollte, Christiansen als wichtigen philosophischen Gesprächspartner zu bezeichnen, wie Steinfeld das tut, sei dahingestellt. Die politischen Überzeugungen der beiden waren in jedem Fall sehr verschieden. Aus Carnaps Tagebüchern geht hervor, dass Christiansen zumindest bis 1935 mit „Deutschlands Erwachen“, also der Machtergreifung der Nationalsozialisten, sympathisierte. 1934 veröffentlichte Christiansen im Selbstverlag das Buch Der neue Gott, nach eigener Aussage die Frucht „,in vielen Jahren gereifter Überzeugungen“. Nach Christiansen ,zeigt sich“ der ,,neue Gott“ oder „,wird erfahren“ in mystischen Erfahrungen des „Heiligen“. Als paradigmatisches Beispiel für dieses „Heilige“ erwähnte Christiansen unter anderen den „Führer“, der dem „Hitlerjungen“ als etwas „Heiliges“ erscheine, ,so lange dieser heroisch glüht“. Dieses und ähnliche Beispiele in Christiansen (1934, S. 166 f.) belegen Christiansens Affinität zum Nazismus. In Carnaps Tagebüchern werden vehemente Auseinandersetzungen zwischen den beiden erwähnt. Nach Steinfeld (2016, S. 15 f.) blieben Carnap und Christiansen jedoch bis zu dessen Tod 1958 in Kontakt.
} 
Lehrbuch der Handschriftendeutung (1933), das in den folgenden zwanzig Jahren mehrere Umarbeitungen und Neuauflagen erlebte. Die Ausgabe von 1947 gibt als Adresse der beiden Autoren ein „Institut für wissenschaftliche Graphologie“ an, als dessen Mitglieder Christiansen sowie Elisabeth Carnap und ihre Tochter Eline Carnap genannt werden. Mit einigem Recht könnte man deshalb behaupten, die Graphologie sei ein Familienunternehmen ${ }^{10}$ der Carnaps gewesen.

\title{
9.4 Graphologische Charakterbeschreibungen
}

\author{
Um die Reichweite und die Grenzen von Carnaps Programm einer Physikalisierung \\ der Graphologie einschätzen zu können, sollen in diesem Abschnitt einige Aspekte \\ typischer graphologischer Charakterbeschreibungen etwas eingehender erörtert \\ werden. Ein von Klages als exemplarisch angesehenes Charakterbild, das in allen \\ Auflagen seines Werkes Handschrift und Charakter unverändert auftaucht, lautet \\ wie folgt:
}

Schreiberin ist eine bewegliche, tätige Persönlichkeit: großzügig, freigesinnt, temperamentvoll, impulsiv und von vielfältigen Gaben des Geistes und des Herzens. Hier: Gemüt, Anteilnahme, Mitgefühl, Hilfsbereitschaft; dort: praktische Klugheit, vielseitige, auch künstlerische Interessen, rasche Auffassungsgabe, Organisationsfähigkeit und nicht zuletzt männlicher Unternehmungsgeist. [...] Es fehlt ihr nicht an schwungvoller Energie, gleichzeitig aber auch nicht an kluger Besonnenheit, die sogar rechnen und einteilen kann.

So scheinen also alle Voraussetzungen $\mathrm{zu}$ harmonischem Leben vorhanden. [...] Dennoch: wer näher zuschaut, bemerkt [...] eine gewisse Leere des Betätigungstriebes; bei aller Rührigkeit einen toten Punkt. Schreiberin selbst wird von dieser Empfindung bisweilen beunruhigt $[\ldots]$

Wo sind nun die Ursachen solcher Unzulänglichkeit zu suchen? In dem allzu männlichen Geist der Schreiberin. Er begabt sie zwar mit mehr als durchschnittlicher Sachlichkeit und Freimütigkeit; aber er nimmt ihr auch jenes allerweiblichste Etwas, dessen wärmende Fülle wir mehr erfühlen als beurteilen können. (Klages 1989 [1917], S. 243)

\footnotetext{
${ }^{10}$ Manche Aktivitäten dieses „Familienunternehmens“ können nur als grenzwertig bezeichnet werden. Nicht nur, dass einige Mitglieder dieser Familie eifrig damit beschäftigt waren, andere Mitglieder graphologisch zu analysieren; in Carnaps Nachlass finden sich überdies mehrere graphologische Analysen von Personen, die (im engeren oder weiteren Sinne) zu Carnaps beruflichem Umfeld gehörten. So hat sich eine ausführliche Analyse Wittgensteins von Elisabeth Carnap erhalten (cf. RC 02-74-10), des weiteren findet sich eine von Christiansen erstellte Analyse einer Freundin von Moritz Schlick („Maja, russisch-jüdische Studentin“), die deutlich von antisemitischen Klischees geprägt ist, welche die Schreiberin als eine Art „slawisch-jüdische Lolita“ charakterisieren: „Im Mittelpunkt der Schrift steht das Herz [...] Es ist ein Kinderherz, so kindhaft prall wie der pralle Körper eines kleinen Kindes. [...] kindhaft, russisch, [...] bewußtes Zigeunertum [...]“" (cf. RC 102-27-05). Es ist deshalb vielleicht nicht ganz abwegig, das verborgene Motiv für Carnaps Projekt der Physikalisierung der Graphologie als einen verzweifelten Versuch zu deuten, dem in seiner Umgebung endemisch verbreiteten graphologischen Unfug doch noch eine rationale Rechtfertigung zu geben. Andererseits beschränkte sich sein Interesse an „Esoterik“ keineswegs nur auf die Graphologie. In seinem Nachlass findet sich ein Beleg, dass er sich von einer Freundin seiner ersten Frau Elisabeth die Handlinien lesen ließ (RC-021-74). Den Hinweis darauf verdanke ich Christian Damböck.
} 
Es geht nicht darum, ob man dieses einer obsoleten Psychologie des frühen 20. Jahrhunderts verpflichtete Charakterbild heute noch für wissenschaftlich hält: Die Rede vom ,allerweiblichsten Etwas und seiner wärmenden Fülle“ dürfte die meisten heutigen Leser wohl etwas befremden. Der Punkt ist, dass Klages’ Beschreibung, auch wenn sie den Charakter der Schreiberin zutreffend wiedergäbe, nie und nimmer aus physikalistischen Beschreibungen von Momentaneigenschaften rekonstruierbar wäre, wie sie Carnap ansatzweise für die Momentaneigenschaft ,,aufgeregt“ skizziert hat.

Die Charakterbilder in Christiansens und Elisabeth Carnaps Lehrbuch der Handschriftendeutung sind von ähnlicher Art wie die in Klages' Handschrift und Charakter, auch wenn die Autoren behaupteten, ihre graphologischen Methoden unterschieden sich wesentlich von denen Klages' (Christiansen und Carnap 1955, S. 13). Amüsant ist die Tatsache, dass Christiansen und Elisabeth Carnap als Objekte ihrer Analysen auch Schriftproben einiger damals prominenter Philosophen genommen haben. So erfährt Heinrich Rickert, Christiansens Doktorvater, im Buch von Christiansen und Elisabeth eine sehr schmeichelhafte Beurteilung:

\begin{abstract}
Sensibilität der Gedanken und Sinne; vollendete Klarheit des Denkens und des Ausdrucks; eine feinsinnige Geistigkeit. Ehrfurcht liegt zugrunde. Der idealistische Aufschwung seiner hohen Oberzeichen wird bestärkt durch das zu Unheimische der zu kurzen Unterlängen (er litt an schwerer Phobie) [...] Leise Müdigkeit in allen Regungen. (Neigung zu dachziegelförmigem Abfall) nötigt ihn zur Ökonomie der Kräfte. Ein hoher Klang echter Noblesse. (Christiansen und Carnap 1955, S. 150)
\end{abstract}

Die ausführlichste Analyse ist Rudolf Carnap selbst vorbehalten, dem eine über mehrere Lebensalter sich erstreckende Darstellung (Christiansen und Carnap 1955, S. 133-135) gewidmet ist: In der Schrift des Siebenjährigen erregt ,die Überfeinheit der Aufstriche“ die Aufmerksamkeit der Graphologin, die ihr zufolge die „Sensibilitätsnot des nervenzarten Kindes“ verrät. Bereits im Schriftbild des Zehnjährigen erblickt ihr graphologisch geschulter Blick ,einen Fanatismus der Genauigkeit, der einige Jahrzehnte später den Weltruf seiner logischen Präzisionsarbeiten begründen wird“. In der Schrift des 18-Jährigen schließlich entdeckt die Graphologin

den Aufbruch des Eigenen zur Freiheit. Die Schrift ist wie erfüllt vom Rausch dieser Freiheit: die neuen Formen flattern noch etwas ungelenk wie Sprünge eines jungen Füllens, [...] etc. etc. (Christiansen und Carnap 1955, S. 134)

Bis heute sind die Charakterbeschreibungen der Graphologie durchweg von ähnlichem Kaliber. Wenn man die Erstellung dieser Art von Texten überhaupt als wissenschaftlich bezeichnen möchte, sind sie Resultate einer Wissenschaft, die wohl zu den letzten zählt, die einer Physikalisierung anheim fielen.

Alle graphologischen Analysen, seien sie von Klages oder von Christiansen und Elisabeth Carnap, enthalten ästhetische, moralische oder sonstwie wertende Begriffe wie „echte Noblesse“, „diplomatische Gewandtheit“, „männlicher Unternehmungsgeist“, „feinsinnige Geistigkeit“. Eine vollständig physikalisierte Graphologie wäre verpflichtet, sie durch rein physikalistische Begriffe zu ersetzen, die nur auf die raumzeitliche ,geometrische“ Gestalt und Dynamik der Schrift Bezug nehmen. Davon kann in sämtlichen graphologischen Charakterbildern von 
Klages oder von Christiansen und Elisabeth Carnap keine Rede sein. Sie alle verwenden ästhetische oder ethische Begriffe, die einen irreduziblen wertenden Teil enthalten, der sich einer physikalistischen Reformulierung entzieht. Die tatsächliche Praxis der Graphologie, selbst wenn man sie als ,,vernünftige“ Praxis akzeptieren würde, ist weit entfernt von jeder Möglichkeit einer Physikalisierung ihrer Begriffe, wie sie Carnap unterstellte.

Carnap haben diese Tatsachen der real existierenden graphologischen Praxis offenbar nicht interessiert. Er setzte kontrafaktisch voraus, dass sie für die Wissenschaftsphilosophie dieser Disziplin keine Rolle spielen. Seine physikalisierte Graphologie, die als Vorbild für eine physikalisierte Psychologie insgesamt dienen sollte, ist also als ein recht „utopisches“ Projekt, was für viele andere Projekte von Carnaps Wissenschaftsphilosophie gilt.

\subsection{Klages in der deutschen Philosophie des frühen 20. Jahrhunderts}

Ludwig Klages war eine der fragwürdigsten Gestalten der deutschen Philosophie in der ersten Hälfte des 20. Jahrhunderts. Es ist sehr wahrscheinlich, dass Carnap schon vor dem Ersten Weltkrieg mit Klages' Werk in Berührung gekommen ist. Carnap war Mitglied des Jenaer „Serakreises“ um den Verleger Eugen Diederichs, in dessen Verlag Klages einige seiner Werke veröffentlich hat. Auch war Carnap auf dem Ersten Freideutschen Jugendtag 1913 auf dem Hohen Meißner, zu dessen „Festschrift“" Klages mit dem Essay Mensch und Erde einen prominenten Beitrag leistete. Allgemein kann man sagen, dass Klages einen erheblichen Einfluss auf Teile der deutschen Jugendbewegung hatte. ${ }^{11}$

Klages hatte in den letzten Jahren der Weimarer Republik einen immensen Einfluss auf die Philosophie und die intellektuelle und kulturelle Sphäre in Deutschland. Sein monumentales Hauptwerk Der Geist als Widersacher der Seele (Klages 1981 [1929-1932]) war ein philosophischer Bestseller. Es kann als Ausarbeitung einer abstrusen dichotomischen Geschichtsmetaphysik verstanden werden, der zufolge die Geschichte der Menschheit in ihrer Gesamtheit eine Auseinandersetzung zwischen den beiden Kräften „Geist“ und „Leben“ darstellt:

Der Geist ,ist“; das Leben vergeht.

Der Geist urteilt; das Leben erlebt.

\footnotetext{
${ }^{11}$ Peter Bernhard hat mich freundlicherweise darauf aufmerksam gemacht, dass Klages selbst, entgegen einem weit verbreiteten Gerücht, nicht an dem Treffen auf dem Hohen Meißner teilgenommen hat, obwohl er das später selbst behauptet hat. Klages' Abwesenheit auf dem Hohen Meißner hat seinem Einfluss auf die deutsche Jugendbewegung aber offenbar keinen Abbruch getan. Bis heute ist Mensch und Erde in zahlreichen Ausgaben immer wieder veröffentlicht worden. Es kann als eine Art Manifest eines radikalen Ökologismus gelten. Klages' Antisemitismus und seine faschistische Ideologie werden von Anhängern der Ökologiebewegung meist ignoriert oder zumindest heruntergespielt.
} 
$[\ldots]$

Das Wesen des „geschichtlichen“ Prozesses der Menschheit (auch „Fortschritt“ genannt) ist der siegreich fortschreitende Kampf des Geistes gegen das Leben mit dem (allerdings nur) logisch absehbaren Ende der Vernichtung des letzteren. (Klages 1981 [1929-1932], S. 69)

Klages’ pathetische Entgegensetzung von „Geist“ und „Leben“ war verquickt mit einem geradezu psychopathischen Antisemitismus, der „den Juden“ zum Repräsentanten des lebensfeindlichen Geistes erkoren hatte:

\begin{abstract}
Alles Menschliche ist dem Juden bloß Gebärde, ja sein menschliches Gesicht selbst ist nur eine Maske. Er ist nicht etwa verlogen, sondern die Lüge selbst. Wir stehen also auf dem Punkt zu entdecken: Der Jude ist überhaupt kein Mensch. Er lebt das Scheinleben einer Larve, die Moloch-Jahwe sich vorband, um auf dem Wege der Täuschung die Menschheit zu vernichten. ${ }^{12}$
\end{abstract}

Klages hat diese Bemerkung bereits 1903 geschrieben; er hielt sie offenbar für so bedeutsam, dass er sie noch vierzig Jahre später sozusagen testamentarisch in dem Werk Rhythmen und Runen (Klages 2013, S. 330) publizierte. Von einer ,jugendlichen Verirrung" lässt sich deshalb kaum sprechen. Judentum und Christentum galten Klages als Repräsentanten des das „Leben“"zerstörenden „Geistes“. Nach Walter Laqueur (1962, S. 34, Fußnote) hatte Klages über viele Jahre hinweg einen beträchtlichen Einfluss auf die deutsche Jugendbewegung. Seine Angriffe auf ein moralisches Gewissen und seine gegen den „Geist“ gerichteten Attacken ebneten in vieler Hinsicht den Weg für eine faschistische Philosophie, auch wenn Klages selbst im nationalsozialistischen Deutschland keine Karriere machte.

Das Merkwürdige ist, dass er Carnap nicht nur als Graphologe beeinflusst hat, sondern ihn darüber hinaus auch philosophisch beeindruckte, obwohl die philosophischen Positionen der beiden eigentlich diametral entgegengesetzt waren - irrationalistische Lebensphilosophie auf der einen, wissenschaftliche und aufklärungsorientierte Philosophie auf der anderen Seite. Diese Entgegensetzung hat aber paradoxerweise nicht dazu geführt, dass Carnap Klages' Graphologie, oder doch den Rest seiner Philosophie, als „,metaphysisch“ kritisiert hätte, obwohl sie noch viel „,metaphysischer“ war als die Hegels, Bergsons oder Heideggers, die Carnap des Öfteren als abzulehnende Beispiele von Metaphysik anführte.

Klages' Philosophie ist von Grund auf dichotomisch angelegt. Genauer gesagt behauptete er:

Leib und Seele sind untrennbar zusammengehörige Pole der Lebenszelle, in die von außenher der Geist, einem Keil vergleichbar, sich einschiebt, mit dem Bestreben, sie untereinander zu entzweien, also den Leib zu entseelen, die Seele zu entleiben und dergestalt endlich alles ihm irgend erreichbare Leben zu ertöten. (Klages 1981 [1929-1932], 7)

\footnotetext{
${ }^{12} \mathrm{Ob}$ der sich hier manifestierende Antisemitismus nun „,metaphorisch“ oder irgendwie anders gemeint war, scheint mir ziemlich belanglos. Natürlich behaupten Klages' moderne Adepten, er wäre kein Nationalsozialist gewesen, da er ja in den 1930er-Jahren aufgrund weltanschaulicher Differenzen mit den Machthabern des „Dritten Reiches“ in Konflikt geraten sei.
} 
Carnap selbst war in seiner gesamten philosophischen Karriere Dichotomien bekanntlich nicht abgeneigt. Das belegen Paare wie „Theorie und Praxis“, „Metaphysik und Wissenschaft“, „Darstellung und Ausdruck“, „Leben und Wissenschaft", „,interne und externe Fragen“ und andere. Auch dem Klages'schen Fundamentalgegensatz von „Geist“ und „Leben“ konnte er anscheinend einiges abgewinnen: In einem Vortrag am Bauhaus in Dessau 1929 konterte er Klages' irrationalistische Invektiven gegen den „Geist“ als ein gegen das „Leben“ gerichtetes Prinzip einigermaßen defensiv mit dem (nicht belegten) Goethe-Zitat „Ist das Leben stark genug, braucht es den Geist nicht zu fürchten“. Diese These gibt Klages insofern Recht, als eine solche Behauptung ja auch einen Antagonismus zwischen „Geist“ und „Leben“ unterstellt, auch wenn sie mit Klages’ pessimistischer Voraussage der Vernichtung des „Lebens“ durch den „Geist“ nicht konform ging.

Carnaps Rezeption von Klages’ Werk lag eine Ingenieursperspektive zugrunde, der zufolge Klages' physikalisierungsaffine Graphologie vom Rest seiner Lehren problemlos getrennt werden könnte. Diese Auffassung, für die Carnap niemals Gründe angegeben hat, widerspricht Klages' eigener Auffassung seines Werkes und wurde auch von der Gemeinde der Graphologen, die Klages als den Gründervater einer modernen, „wissenschaftlichen“ Graphologie ansahen, nicht geteilt. So weist der Herausgeber der 29. Auflage von Klages' Handschrift und Charakter, Bernhard Wittlich, in seinem „Geleitwort“ darauf hin, dass die „Wurzel des Lebenswerkes von Klages seine erste graphologische Abhandlung" (eben Handschrift und Charakter, 1910) gewesen sei, und die Krönung Der Geist als Widersacher der Seele (1981 [1929-1932]), auch wenn dieser Zusammenhang von Graphologie und Metaphysik einer unparteiischen Anerkennung von Klages' graphologischen Verdiensten nicht immer zuträglich gewesen sei:

Gerade weil als Wurzel des Lebenswerkes von Klages seine erste graphologische Abhandlung anzusehen ist und als dessen Krönung sein philosophisches Hauptwerk Der Geist als Widersacher der Seele, ergaben sich infolge der engen Verflechtung seiner Ausdruckskunde mit seiner Metaphysik mancherlei Angriffsflächen für philosophisch und weltanschaulich anders eingestellte Kritiker. (Wittlich 1989, X)

Diese enge Verflechtung von Graphologie, ,Ausdruckskunde“ und Metaphysik bei Klages hat für Carnap keine Rolle gespielt. Er nahm Klages’ Graphologie als etwas, das vom Rest seines philosophischen Euvres ohne Probleme separierbar sei.

\subsection{Cassirers Kritik an Carnaps Physikalismus}

Etwa um dieselbe Zeit, als Carnap versuchte, Klages' „ausdruckswissenschaftliche" Graphologie für die Physikalisierung der Psychologie in Dienst zu nehmen, unternahm es Cassirer, den Ort von Klages' Denken in der Landschaft der damaligen deutschen Philosophie genauer zu beschreiben. Er sah Carnaps Logischen Empirismus und Klages' Lebensphilosophie als Gegenpole an, weil sie die Rolle der Ausdrucksfunktion für die Konstitution der Welt absolut gegensätzlich 
einschätzten. Genauer gesagt betrachtete er die beiden als in entgegengesetzte Richtungen weisende Fehlentwicklungen. Carnap war für ihn ein Theoretiker, der der Ausdrucksfunktion jegliche objektive Bedeutung absprach, Klages hingegen ein Mystagoge, dem Ausdruck alles bedeutete, Darstellung hingegen nichts. Klages' metaphysische Philosophie des durch den „Geist“ tödlich bedrohten „Lebens“ war für Cassirer ein durch Ausdrucksbesessenheit gekennzeichneter Ansatz. Für einen solchen Ansatz war der erlebte Ausdruck alles, jede objektive Darstellung eine verfälschende Illusion. Für Carnap hingegen war Ausdruck höchstens ein kognitiv irrelevantes Beiwerk einer kognitiven Unternehmung. Cassirers „Kritische Philosophie" plädierte für einen vermittelnden Standpunkt, was die Einschätzung der Leistung der Ausdrucksfunktion anging:

Unser Standpunkt ist der ,kritische“ - nicht Falschheit (Skepsis) oder Wahrheit (Metaphysik) der Ausdrucksfunktion, sondern kritische „Begrenzung“: kritische Begrenzung und kritische Rechtfertigung ihrer Leistung: Aufbau der Kulturwelt. (Cassirer 1995, ECN1, S. 121)

Die „kritische Begrenzung“ und „kritische Rechtfertigung“ der Ausdrucksfunktion für den Aufbau der Kulturwelt, wozu auch der Aufbau der wissenschaftlichen Welt gehörte, war nicht mit einer dogmatisch zu setzenden These der Philosophie zu erledigen, sondern erforderte eine phänomenologische Aufweisung, wie Cassirer es im Anschluss an Husserl ausdrückte. Grundlage einer solchen Aufweisung war die Einsicht, dass die physikalische Welt keineswegs die Grundlage ist, auf der alles andere beruhte, sondern nur eine Konsequenz der „Lebenswelt“. Das, so Cassirer, lasse sich zwar nicht deduktiv beweisen, aber durch eine phänomenologische Analyse aufweisen. Der eigentliche Fehler eines strikten Physikalismus bestand für ihn in der Annahme, ursprünglich wäre nur „Physisches“ gegeben. Wer von dieser These ausgehe, sei mit dem letzten Endes unlösbaren Problem konfrontiert, zu erklären, wie dieses Physische sich in ein „Psychisches“ verwandeln könne. Das betreffe insbesondere die Existenz des „Fremdpsychischen“, die der Physikalismus ja schlechthin bestreitet:

Der strenge „Physikalismus“ erklärt nicht nur alle Beweise, die man für die Existenz des „Fremdpsychischen“ zu geben versucht hat, für unzulänglich oder ungültig, sondern er leugnet auch, dass man nach einem solchen Fremdpsychischen, nach einer Welt, nicht des „Es“, sondern des „Du“, mit Sinn fragen kann. Nicht nur die Antwort, sondern schon die Frage ist mythisch, nicht philosophisch, und sie muss daher radikal ausgemerzt werden.

Diese physikalistische Position sei genetisch und phänomenologisch nicht haltbar. Die gemeinsame Welt - die Welt des Ich und Du - ist früher als die Welt der Dinge - die Welt des Es. Zum Es gelange man nur über das Du. Ein radikaler Physikalismus, der die Dingwelt an die erste Stelle setzt, begehe den Irrtum eines hysteron proteron. Das lasse sich nur vermeiden, wenn man den Begriff des Physischen nicht dogmatisch, sondern transzendental versteht. Tue man diesen Schritt,

so finden wir ja, daß die sogenannte >physische< Welt nichts anderes ist als der Inhalt der Erfahrung, sofern vorausgesetzt wird, daß er nicht nur ,mir selbst“ angehört, nicht nur für mich in diesem meinen >Hier $<$ und >Jetzt $<$ gegeben ist, sondern daß er 1) für mich >immer in der gleichen Weise vorhanden ist, [... 2) für alle andern wahrnehmenden, denkenden Subjekte denselben Zug [...] zeigt [...] - diese Hypothesis eines koinos kosmos für alle 
Subjekte: dies ist ein notwendiger, integrierender konstitutiver Bestandteil des Begriffs >Erfahrungswelt< oder, was auf dasselbe hinausläuft, des Begriffs >Physische Welt<. (Cassirer 2011, EK4, S. 153 ff.)

Der Ausgangspunkt der Physik sei deshalb, so Cassirer, nicht das Physische, sondern die Erkenntnis des Physischen, die immer schon einen koinos kosmos voraussetzt, der von vornherein als ,gemeinsame Welt“ der Erkennenden konstituiert ist. Cassirer attestierte Carnap, dies im Logischen Aufbau der Welt zumindest in Ansätzen auch erkannt zu haben. Ihm zufolge stimmten „kritische“ Philosophie, Phänomenologie und die Konstitutionsanalyse Carnaps in der Grundeinsicht überein, daß das Physische nicht unmittelbar gegeben sei. Erst später, in seiner physikalistischen Post-Aufbau-Phase, habe Carnap diese ursprüngliche Einsicht offenbar aufgegeben.

Cassirers phänomenologische Konstitution einer umfassenden personalen und dinglichen Welt ist also gekennzeichnet durch zwei wesentlich verschiedene Konstitutionsverfahren, die nicht aufeinander reduzierbar sind. Diese Irreduzibilität, so gab Cassirer explizit zu, war nicht logisch beweisbar, sondern nur genetisch und phänomenologisch aufweisbar. Sie beruhte, was hier nicht im Einzelnen erörtert werden soll, auf zwei verschiedenen Arten der Wahrnehmung, nämlich der Dingwahrnehmung und der Ausdruckswahrnehmung, die beide gleich ursprünglich sind. Eine Konsequenz dieser Irreduzibilität verschiedener Konstitutionsverfahren ist, dass eine physikalistische Sprache nicht die einzige Sprache sein kann, die die Philosophie zur Kenntnis nehmen sollte:

Wir müssen, ohne Vorbehalt und erkenntnistheoretisches Dogma, jede Art von Sprache, die wissenschaftliche Sprache, die Sprache der Kunst, der Religion, usf., in ihrer Eigenart zu verstehen suchen; wir müssen bestimmen, wieviel sie zum Aufbau einer >gemeinsamen Welt< beiträgt. (Cassirer 2011, EK4, S. 42)

Cassirer ist auf Carnaps Projekt einer physikalisierten Graphologie als Avantgarde einer vollständig physikalisierten Psychologie (cf. Carnap 1932/1933) nie eingegangen. Aus seiner Konzeption einer umfassenden Konstitutionstheorie, die eine gemeinsame, durch Ausdruckswahrnehmung und Dingwahrnehmung charakterisierte Welt intendierte, lässt sich jedoch erschließen, dass er Carnaps Graphologieprojekt keine sehr großen Sympathien entgegengebracht hätte.

\subsection{Physikalistische Graphologie als wissenschaftsphilosophisches Artefakt}

Meines Wissens hat niemand Carnaps Skizze einer „physikalisierten Graphologie“ als Beleg für die Physikalisierbarkeit der Psychologie und damit der Wissenschaft insgesamt jemals ernst genommen. Weder sind ihm die Graphologen gefolgt und haben die Physikalisierung ihrer "Wissenschaft" ernsthaft in Angriff genommen, noch ist Carnaps Idee bei Psychologen und (Wissenschafts-)Philosophen auf Interesse oder gar Zustimmung gestoßen. Carnap selbst ist in späteren Arbeiten nie 
mehr auf das Thema Graphologie zurückgekommen. Das heißt allerdings nicht, dass sich auch das Thema „Physikalismus“ für ihn erledigt hätte. ${ }^{13}$

Zwar taucht das Projekt einer Physikalisierung der Graphologie nach 1932 in Carnaps Agenda nicht mehr auf, kann es als typisch für Carnaps reduktionistische Konzeption von Wissenschaftsphilosophie angesehen werden. Es beruhte wesentlich auf einer sauberen Separierung von Praxis und Theorie, von Metaphysik und Physik, von Ausdruck und Darstellung usw. Funktional analoge Separierungen finden sich Klages: Geist und Leben, Gesetz und Willkür, Nähe und Ferne, Wirklichkeit und Bilder etc. Auch wenn also Carnaps und Klages' Gedankengebäude inhaltlich kaum etwas gemeinsam haben, teilen sie eine Vorliebe für strikte Dichotomisierungen. Während Carnap im Laufe seiner philosophischen Karriere eine Reihe solcher Dichotomien propagierte, hat Klages letztlich alles unter den absurden Antagonismus von „Geist“ und „Seele“" subsumiert.

Der in Carnaps gesamtem Werk sich durchhaltende dichotomische Charakter seines Denkens findet eine frühe Ausprägung in der Graphologie-Episode. Auch wenn dies also Episode geblieben ist und keine direkten Spuren in Carnaps späterem Werk hinterlassen hat, sollte man sie nicht als belanglos für Carnaps Wissenschaftsphilosophie abtun. Im Gegenteil, man kann sie als typisch betrachten für manche anderen Versuche Carnaps, die sperrige Wirklichkeit der real existierenden Wissenschaften in formale Schemata einer allzu logischen und formalen Wissenschaftsphilosophie einzupassen, womit seine Wissenschaftsphilosophie nicht selten in die Gefahr geriet, sich anstatt mit der wirklichen Wissenschaft mit einer logischen Fiktion zu befassen.

\section{Literatur}

Carnap, R. 1931. Die physikalische Sprache als Universalsprache der Wissenschaft. Erkenntnis 2:432-465.

. 1932/1933. Psychologie in physikalischer Sprache. Erkenntnis 3:107-142.

. 1963. Replies. In The philosophy of Rudolf Carnap, Hrsg. P.A. Schilpp, 859-1012. LaSalle/Chicago: Open Court.

Cassirer, E. 1995. Zur Metaphysik der symbolischen Formen. In: ders., Nachgelassene Manuskripte und Texte, Bd 1 (ECN 1). Hamburg: Meiner.

—. 2011. Symbolische Prägnanz, Ausdrucksphänomen und „Wiener Kreis“. In: ders., Nachgelassene Manuskripte und Texte, Bd 4 (ECN 4). Hamburg: Meiner.

Christiansen, B. 1934. Der neue Gott. Buchenbach: Felsen.

Christiansen, B., und E. Carnap. 1955 (1933). Lehrbuch der Handschriftendeutung. Stuttgart: Reclam. Neu bearb. u. ersch. als: Lehrbuch der Graphologie. Stuttgart: Reclam.

Freud, S. 1917. Eine Schwierigkeit der Psychoanalyse. Imago: Zu Anwendungen der Psychoanalyse auf die Geisteswissenschaften 5:1-7.

— 1940. Abriß der Psychoanalyse. Internationale Zeitschrift für Psychoanalyse-Imago 25 (1): 7-67.

\footnotetext{
${ }^{13}$ Noch in den „Replies“ in dem seiner Philosophie gewidmeten Schilpp-Band geht er ausführlich auf das Thema „Physikalismus“ ein (cf. Carnap 1963, S. 857 ff.).
} 
Habermas, J., 2001, Erkenntnis und Interesse, Frankfurt/Main, Suhrkamp Verlag.

Kim, J., 2008, Physicalism, or something near enough, Princeton/NJ, Princeton University Press. Klages, L. 1981 [1929-1932]. Der Geist als Widersacher der Seele. Bonn: Bouvier.

1989 [1917]. Handschrift und Charakter: Gemeinverständlicher Abriß der graphologischen Technik. Für die Deutungspraxis bearbeitet und ergänzt von Bernhard Wittlich, 29. Aufl. Bonn: Bouvier. 2013 [1913]. Mensch und Erde. Berlin: Matthes \& Seitz.

Schilpp P.A. Hrsg. 1963 [1997]. The philosophy of Rudolf Carnap. LaSalle/Chicago: Open Court. Steinfeld, T. 2016. Ich will, ich kann: Moderne und Selbstoptimierung. Konstanz: Konstanz University Press.

Thomsen, A., Hrsg. 2008. Wer war Dr. Broder Christiansen? Leben und Wirkung eines deutschen Philosophen. Neukirchen: Verl Make a Book.

Wittlich, B., 1989, Geleitwort, in L. Klages, Handschrift und Charakter. Gemeinverständlicher Abriß der graphologischen Technik, Bonn, Bouvier Verlag.

Open Access This chapter is licensed under the terms of the Creative Commons Attribution 4.0 International License (http://creativecommons.org/licenses/by/4.0/), which permits use, sharing, adaptation, distribution and reproduction in any medium or format, as long as you give appropriate credit to the original author(s) and the source, provide a link to the Creative Commons license and indicate if changes were made.

The images or other third party material in this chapter are included in the chapter's Creative Commons license, unless indicated otherwise in a credit line to the material. If material is not included in the chapter's Creative Commons license and your intended use is not permitted by statutory regulation or exceeds the permitted use, you will need to obtain permission directly from the copyright holder.

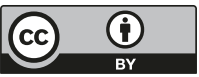

